\title{
Qualidade do sono e fadiga em mulheres com câncer de mama durante o tratamento quimioterápico
}

Sleep quality and fatigue in women with breast cancer during chemotherapy

Calidad del sueño y fatiga en mujeres com cáncer de mama durante el tratamiento quimioterápico

\section{Pamina Roberta da Silva', Lóris Aparecida Prado da Cruz ${ }^{\text {II }}$, Talita Garcia do Nascimento ${ }^{I I I}$ Thais de Oliveira Gozzo ${ }^{\text {IV }}$}

\begin{abstract}
Resumo: Objetivo: identificar a ocorrência de alterações no sono antes, durante e após o tratamento quimioterápico e correlacionar as alterações do sono com episódios de fadiga em mulheres com câncer de mama. Método: participaram do estudo 26 mulheres seguidas no Ambulatório de Mastologia de um hospital universitário que responderam o Pittsburgh Sleep Quality Index e a Functional Assessment of Cancer Therapy Fatigue para avaliar o sono e a fadiga respectivamente, em três momentos. Resultados: as participantes apresentaram distúrbios do sono, ao longo do tratamento, caracterizados por calor noturno e dificuldade em iniciar o sono e que influenciaram na piora da qualidade do mesmo. Houve correlação entre qualidade do sono e fadiga, sendo que a má qualidade do sono aumentou os episódios de fadiga. Conclusão: a identificação da ocorrência destes eventos durante a quimioterapia favorece que a equipe profissional realize orientações para manejo adequado dos eventos adversos.
\end{abstract}

Descritores: Enfermagem; Neoplasias da mama; Quimioterapia Combinada; Sono; Fadiga

\begin{abstract}
Objective: To identify the occurrence of changes in sleep before, during and after chemotherapy treatment and to correlate sleep disorders with episodes of fatigue in women with breast cancer. Method: Twentysix women followed at the Mastology Outpatient Clinic of a university hospital who responded to the Pittsburgh Sleep Quality Index and to the Functional Assessment of Cancer Therapy Fatigue to assess sleep and fatigue, respectively, in three moments. Results: The participants presented sleep disturbances throughout the treatment, characterized by night heat and difficulty to start sleep and that influenced the quality of sleep. There

\footnotetext{
I Enfermeira. Mestre em Ciências pelo Programa de Pós-Graduação em Saúde Pública da Escola de Enfermagem de Ribeirão Preto da Universidade de São Paulo. Ribeirão Preto-SP, Brasil. E-mail: paminaroberta@hotmail.com ORCID https://orcid.org/0000-0002-7705-7879

II Enfermeira. Doutoranda pelo Programa de Pós-Graduação em Saúde Pública da Escola de Enfermagem de Ribeirão Preto da Universidade de São Paulo. Ribeirão Preto-SP, Brasil. E-mail: loris.pradodacruz@gmail.com ORCID https://orcid.org/0000-0003-2474-8921

III Enfermeira. Doutora em Ciências pelo Programa de Pós-Graduação em Saúde Pública da Escola de Enfermagem de Ribeirão Preto da Universidade de São Paulo. Ribeirão Preto-SP, Brasil. E-mail: talitagarcian@gmail.com ORCID https://orcid.org/0000-0002-1758-1628

IV Enfermeira. Professora Associada da Escola de Enfermagem de Ribeirão Preto da Universidade de São Paulo. Ribeirão Preto-SP, Brasil. Email: thaisog@eerp.usp.br ORCID https://orcid.org/0000-0002-7687-9459
} 
Qualidade do sono e fadiga em mulheres com câncer de mama durante o tratamento quimioterápico | 2

was a correlation between sleep quality and fatigue, with poor sleep quality increasing episodes of fatigue. Conclusion: Identifying the occurrence of these events during chemotherapy helps the professional team to provide guidelines for an adequate management of adverse events.

Descriptors: Nursing; Breast neoplasms; Drug therapy, combination; Sleep; Fatigue

Resumen: Objetivo: identificar la manifestación de cambios en el sueño antes, durante y después de la quimioterapia y correlacionar las alteraciones del sueño con episodios de fatiga en mujeres con cáncer de mama. Método: participaron del estudio 26 mujeres controladas en el Ambulatorio de Mastología de un hospital universitario que respondieron el Pittsburgh Sleep QualityIndex y la Functional Assessment of Cancer Therapy Fatigue para evaluar el sueño y la fatiga respectivamente, en tres momentos. Resultados: las participantes presentaron trastornos del sueño, a lo largo del tratamiento, caracterizados por calor nocturno y dificultad para iniciar el sueño, trastornos que ejercieron un efecto adverso sobre la calidad del sueño. Se registró una correlación entre la calidad del sueño y la fatiga, siendo que la mala calidad del sueño aumentó la cantidad de episodios de fatiga. Conclusión: identificar la manifestación de estos eventos durante la quimioterapia ayuda a que el equipo profesional realice orientaciones para el manejo adecuado de los eventos adversos.

Descriptores: Enfermería; Neoplasias de la mama; Quimioterapia combinada; Sueño; Fatiga

\section{Introdução}

A quimioterapia é uma das modalidades terapêuticas empregadas no controle do câncer e seus eventos adversos atingem diferentes aspectos da vida dos indivíduos, uma vez que afetam as esferas biopsicossociais e geram sérios desconfortos como alterações emocionais, náuseas, vômitos, distúrbios do sono e fadiga. ${ }^{1}$

Os distúrbios do sono e a fadiga são frequentemente classificados como os eventos adversos mais impactantes e debilitantes do tratamento do câncer, apesar de suas taxas serem difíceis de estimar, devido as diferentes medidas utilizadas e pela falta de especificidade clínica. ${ }^{2}$

A dificuldade na manutenção do sono pode ser vinculada ao diagnóstico, ao estresse, a ansiedade ou a outros sintomas relacionados ao próprio tratamento como a dor, a náusea e a até mesmo a própria fadiga. Ressalta-se a possibilidade de esta ocorrer antes, durante e até cinco anos após o término do tratamento quimioterápico, e também ser associada a depressão e a dor..$^{3-4}$

Contudo, a preocupação é intensificada nas mulheres com câncer de mama. Estudos mostram que a fadiga e os distúrbios do sono são os sintomas mais relatados por mulheres tratadas para câncer de mama inicial e dentre estes, a fadiga estava entre os mais angustiantes 
em pacientes em quimioterapia. ${ }^{5}$ Além disso, essas mulheres apresentam humor mais deprimido e depressão após seis meses do término do tratamento, asseverando a magnitude dos efeitos da quimioterapia e a importância da sua identificação e manejo adequado. ${ }^{6-7}$

A fadiga pode aumentar com o início do tratamento, porém, não aumenta com o tempo. Durante a quimioterapia, a frequência e a duração do despertar noturno e a dificuldade em adormecer aumentam, e relatos demostraram a pior qualidade do sono na primeira noite após o tratamento. $^{8}$

Foram estudados os possíveis antecedentes e desfechos de fadiga e distúrbios do sono durante a quimioterapia. Embora a idade não tenha sido relatada como relacionada à fadiga durante a quimioterapia, as mulheres adultas mais velhas apresentaram piora na qualidade do sono; a renda e o estágio da doença não foram relacionados à fadiga ou distúrbios do sono durante o tratamento. Além disso, foi observado que fadiga e baixa qualidade do sono podem predizer diminuição no nível de atividade durante a quimioterapia para câncer de mama. ${ }^{8}$

Deste modo, levando-se em consideração que, quanto mais se conhece a respeito de um sintoma, melhor poderá ser a assistência, questiona-se: qual a relação entre as alterações na qualidade do sono e a ocorrência de fadiga entre as mulheres com câncer de mama que estão em tratamento quimioterápico? Assim, este estudo teve como objetivo identificar a ocorrência de alterações no sono antes, durante e após o tratamento quimioterápico e correlacionar as alterações do sono com episódios de fadiga em mulheres com câncer de mama.

\section{Método}

Estudo analítico e longitudinal, desenvolvido no Ambulatório de Mastologia de um hospital universitário do estado de São Paulo, no período de junho de 2012 a janeiro de 2013. A população do estudo foi composta por mulheres que receberam o diagnóstico de câncer de mama e tinham a indicação de tratamento quimioterápico. Foram considerados critérios de inclusão mulheres com idade igual ou superior a 18 anos e que iniciariam o tratamento 
Qualidade do sono e fadiga em mulheres com câncer de mama durante o tratamento quimioterápico | 4 quimioterápico neoadjuvante ou adjuvante pela primeira vez. Como critérios de exclusão considerou-se mulheres que não podiam se expressar individualmente; com dificuldade de leitura e de compreensão; com doença metastática ou outro tipo de tumor maligno no diagnóstico do câncer de mama e mulheres no ciclo gravídico-puerperal.

Realizou-se o cálculo amostral a partir de uma estimativa de 100 casos novos/ano de câncer de mama atendidos no referido ambulatório, por meio da seguinte fórmula: ${ }^{9}$

$$
n=\frac{(Z \alpha / 2)^{2} \times p \times q}{E^{2}} \quad \mathrm{n}=\underline{(1,96)^{2} \times 0,5 \times 0,5}=24
$$

$\mathrm{Na}$ referida fórmula, os valores de $n$ foram calculados de maneira que o erro cometido na estimativa de proporções não ultrapasse o valor $E$, com probabilidade P. O valor para $p$ e $q$ foi de 0,50, de maneira a garantir que $E$ seja o máximo valor possível do erro. Já o componente $(Z \alpha / 2)$ é o valor da curva normal que corresponde à probabilidade $\mathrm{P}$ desejada. Dessa maneira, com $\mathrm{P}=$ 95\% que corresponde a 1,96 na distribuição normal e $E=20 \%$, obteve-se um $n=24$ participantes para este estudo. Entretanto, pela possibilidade de perda de participantes durante o seguimento, acresceu-se $10 \%$ ao $n$ final, totalizando 26 participantes incluídas.

Para a coleta de dados, as participantes responderam a um instrumento com dados acerca da caracterização sociodemográfica, informações clínicas e do tratamento proposto. Estes foram obtidos em entrevista com a participante anteriormente ao tratamento quimioterápico (T0) e por meio de revisão do prontuário médico. Para avaliação do sono e da fadiga foram utilizados instrumentos específicos, aplicados em T0, na metade do tratamento (T1) e após o último ciclo do mesmo (T2).

Para avaliar a fadiga foi aplicada a escala Functional Assessment of Cancer Therapy Fatigue (FACT - F) versão 4. A FACT-F foi elaborada, especificamente, para medir a fadiga em indivíduos com câncer e validada com pacientes americanos e está validada para o uso no 
Brasil..10-11 Neste estudo, o uso da escala foi autorizado pelos autores, os quais também disponibilizaram as instruções para a aplicação e análise dos dados.

A FACT-F é composta por 13 itens e cada um possui uma escala do tipo Likert de cinco pontos, os quais seguem o padrão: "Nem um pouco", "Um pouco", "Mais ou menos", "Muito", "Muitíssimo". As mulheres foram orientadas a responder um item por questão referentemente aos últimos sete dias. A pontuação final da FACT-F é obtida pela soma dos escores, e pode variar de 0 a 52 pontos, sendo que quanto maior o número de pontos menor a ocorrência de fadiga. ${ }^{10-11}$

A avaliação os aspectos relacionados ao sono, foi realizada com a aplicação do Pittsburgh Sleep Quality Index (PSQI), que é o padrão ouro e a medida mais utilizada para avaliação da qualidade do sono em várias populações em estudos de pesquisa. O PSQI mensura a qualidade subjetiva do sono e a ocorrência de seus distúrbios ao longo do mês anterior à aplicação. ${ }^{12} \mathrm{O}$ PSQI foi traduzido e validado para o Brasil. ${ }^{13}$

O questionário é composto por 19 questões, abertas e fechadas, agrupadas em sete componentes: qualidade subjetiva do sono, duração, latência, eficiência, distúrbios do sono, uso de medicamentos para dormir e disfunção diurna. Cada componente recebe uma pontuação estabelecida de zero a três pontos, e o escore global varia de 0 a 21 pontos. Os escores superiores a cinco, indicam má qualidade do sono. ${ }^{13}$

A análise dos dados foi realizada por meio de estatística descritiva com médias, medianas e desvio padrão das variáveis de interesse. Para a comparação dos resultados, foram utilizados coeficientes de correlação de Spearman e respectivos testes de significância para avaliar as possíveis correlações entre os escores da subescala fadiga e PSQI; e Teste de Mann-Whitney para comparar a magnitude dos escores de cada escala em duas classes da qualidade do sono. Também foi aplicado o alpha de Crombach para avaliar a consistência interna das respostas às escalas e ao instrumento. ${ }^{9}$ 
Qualidade do sono e fadiga em mulheres com câncer de mama durante o tratamento quimioterápico I 6

Este estudo foi aprovado pelo Comitê de Ética em Pesquisa em 01 de junho de 2011 (Protocolo número 1302/2011). A pesquisa foi conduzida respeitando os princípios éticos exigidos.

\section{Resultados}

A faixa etária das participantes variou de 27 a 68 anos, a maioria $(53,9 \%)$ estava na faixa etária de 31 a 50 anos, a média de idade foi de 48 anos (DP: 11,7). Das participantes, 65,4\% possuíam companheiro; 57,7\% referiram ter de 3 a 10 anos de estudo (Tabela 1).

Em relação às comorbidades, $65,3 \%$ relataram pelo menos uma, sendo que $34,6 \%$ relataram apresentar hipertensão arterial sistêmica. Quanto ao uso de medicamentos, 57,7\% referiram fazer uso regular de um ou mais, sendo os anti-hipertensivos, antidiabéticos e antidepressivos os mais relatados; $61,5 \%$ estavam na pré-menopausa e $73,1 \%$ não realizavam atividade física (Tabela 1).

Tabela 1- Distribuição das mulheres submetidas à quimioterapia para câncer de mama, segundo idade, estado civil, anos de estudo, comorbidades, uso de medicações, prática de atividade física e menopausa. Ribeirão Preto (SP), 2013.

\begin{tabular}{ccc}
\hline Variável & $\mathrm{N}$ & $\mathbf{\%}$ \\
\hline Idade & & \\
$\leq 30$ & 01 & 3,8 \\
31 a 40 & 06 & 23,1 \\
41 a 50 & 08 & 30,8 \\
51 a 60 & 06 & 23,1 \\
61 a 68 & 05 & 19,2 \\
Estado civil & & \\
Com companheiro & 17 & 65,4 \\
Sem companheiro & 09 & 34,6 \\
Anos de estudo & & \\
3 a 10 anos & 15 & 57,7 \\
11 a 15 anos & 11 & 42,3 \\
Comorbidades & & \\
Sem comorbidades & 09 & 34,7 \\
Com comorbidades & 17 & 65,3 \\
Uso de medicamentos & & \\
\hline
\end{tabular}


7 I Silva PR, Lóris Cruz AP, Nascimento TG, Gozzo TO

\begin{tabular}{ccc}
\hline Sim & 15 & 57,7 \\
Não & 11 & 42,3 \\
Status menopausal & & \\
Pré-menopausa & 16 & 61,5 \\
Pós-menopausa & 10 & 38,5 \\
Prática atividade física & & \\
Sim & 07 & 26,9 \\
Não & 19 & 73,1 \\
\hline
\end{tabular}

Fonte: Banco de dados da pesquisa

Todas as participantes foram submetidas ao tratamento cirúrgico, sendo que dessas, $87,5 \%$ realizaram cirurgia conservadora, e a mais frequente foi a tumorectomia. Dentre as participantes, $38,5 \%$ realizaram quimioterapia neoadjuvante e $61,5 \%$ adjuvante. Os protocolos utilizados foram o FEC (Fluouracil; Epirrubicina e Ciclofosfamida), EC-T (Epirrubicina, Ciclofosfamida e Docetaxel), EC-TH (Epirrubicina, Ciclofosfamida, Docetaxel e Trastuzumab) e TC-H (Docetaxel, Ciclofosfamida e Trastuzumab).

A má qualidade do sono estimada pelo escore global do PSQI-BR, foi de 42,3\% em T0, 34,6\% em T1 e de 57,5\% em T2. A análise de confiabilidade do PSQI-BR apresentou resultados satisfatórios para o alpha de Crombach nos três tempos: T0- 0,73; T1- 0,80; T2- 0,76.

A qualidade do sono foi associada às variáveis sociodemográficas e clínicas (idade; anos de estudo; situação atual de emprego; estado civil; exercício físico; comorbidades; uso de medicações; menopausa; esquema quimioterápico; tipo de quimioterapia; duração da quimioterapia e tipo de cirurgia) nas três aplicações do questionário. Observou-se escore estatisticamente significante somente para o estado civil, nos três momentos, dado que confirma a importância deste aspecto para a qualidade do sono das mulheres com câncer de mama (Tabela 2).

Tabela 2- Distribuição das mulheres submetidas a quimioterapia para o câncer de mama segundo a qualidade do sono em relação à variável estado civil. Ribeirão Preto (SP), 2013.

\begin{tabular}{ccccc}
\hline PSQI & Com companheiro & Sem companheiro & Total & Valor de $\mathbf{p}$ \\
\hline Sono bom $(<8)$ & $\mathbf{1 3}$ & 02 & 15 & \\
\hline
\end{tabular}


Qualidade do sono e fadiga em mulheres com câncer de mama durante o tratamento quimioterápico | 8

\begin{tabular}{cccccc}
\hline T0 & Sono ruim $(\geq 8)$ & 04 & $\mathbf{0 7}$ & 11 & $\mathbf{0 , 0 1 4}$ \\
& Total & 17 & 09 & 26 & \\
& Sono bom $(<8)$ & $\mathbf{1 4}$ & 03 & 17 & \\
$\mathbf{T 1}$ & Sono ruim $(\geq 8)$ & 03 & $\mathbf{0 6}$ & 09 & $\mathbf{0 , 0 2 8}$ \\
& Total & 17 & 09 & 26 & \\
& Sono bom $(<8)$ & $\mathbf{1 1}$ & 0 & 11 & \\
$\mathbf{T}$ & Sono ruim $(\geq 8)$ & 06 & $\mathbf{0 9}$ & 15 & $\mathbf{0 , 0 0 2}$ \\
& Total & 17 & 09 & 26 & \\
\hline
\end{tabular}

Fonte: Banco de dados da pesquisa

$\mathrm{P}<0,05$ pelo Teste Exato de Fisher

Nas quatro questões abertas do instrumento PSQI que são relacionadas à quantidade de sono por noite, foi mais frequente nas três aplicações, ir para cama após às 22 horas, levar até 40 minutos para pegar no sono, despertar entre seis e oito horas da manhã e dormir de sete a nove horas por noite. Estes dados sugerem ausência de alterações nos aspectos quantitativos do sono no decorrer da quimioterapia, entretanto, algumas mulheres relataram problemas que interferiram no início e na continuidade do sono durante o tratamento que foi qualificado, principalmente, por não conseguir dormir em menos de trinta minutos $(\mathrm{T} 0=38,5 \%, \mathrm{~T} 1=38,5 \%$, $\mathrm{T} 2=50 \%)$, despertar durante a noite para ir ao banheiro ( $\mathrm{T} 0=53,8 \%$; $\mathrm{T} 1=50 \%$, $\mathrm{T} 2=57,7 \%)$ e queixas de calor noturno $(\mathrm{T} 0=30,8 \%, \mathrm{~T} 1=46,1 \%, \mathrm{~T} 2=61,5 \%)$, com frequência de três ou mais vezes por semana (Tabela 3).

Tabela 3- Distribuição das mulheres submetidas à quimioterapia para câncer de mama segundo os distúrbios do sono do instrumento PSQI. Ribeirão Preto (SP), 2013.

\begin{tabular}{cccc}
\hline Distúrbios do Sono & T0 & T1 & T2 \\
& N (\%) & N (\%) & N (\%) \\
\hline $\begin{array}{c}\text { Não conseguiu pegar no sono em menos } \\
\text { de trinta minutos }\end{array}$ & $10(38,5 \%)$ & $10(38,5 \%)$ & $13(50 \%)$ \\
Acordou no meio da noite ou de manhã & $09(34,6 \%)$ & $13(50 \%)$ & $11(42,3 \%)$ \\
mais cedo do que de costume & & & \\
Teve que se levantar para ir ao banheiro & $14(53,8 \%)$ & $13(50 \%)$ & $15(57,7 \%)$ \\
Teve dificuldade para respirar & $02(7,6 \%)$ & $01(3,9 \%)$ & $02(7,6 \%)$ \\
Tossiu ou roncou alto & $09(34,6 \%)$ & $04(15,4 \%)$ & $09(34,6 \%)$ \\
\hline
\end{tabular}


9 I Silva PR, Lóris Cruz AP, Nascimento TG, Gozzo TO

\begin{tabular}{cccc}
\hline Sentiu muito frio & $04(15,4 \%)$ & $05(19,2 \%)$ & $07(26,9 \%)$ \\
Sentiu muito calor & $08(30,8 \%)$ & $12(46,1 \%)$ & $16(61,5 \%)$ \\
Teve pesadelos & $01(3,9 \%)$ & $04(15,4 \%)$ & $01(3,9 \%)$ \\
\hline
\end{tabular}

Fonte: Banco de dados da pesquisa

A FACT-F apresentou boa confiabilidade interna nos três momentos de coleta dos dados, com alpha de Crombach de 0,85 no T0; 0,85 no T1; e de 0,83, no T2. Os escores máximos e mínimos não apresentaram grande variação ao longo do estudo (Tabela 4).

Tabela 4- Distribuição das mulheres submetidas à quimioterapia para câncer de mama, segundo os escores máximos, mínimos, média e desvio padrão para a FACT-F, nas três fases da coleta T0, T1 e T2. Ribeirão Preto (SP), 2013.

\begin{tabular}{ccccc}
\hline FACIT-F $(\mathbf{0 - 5 2})$ & Máx. & Mín. & MD & DP \\
\hline T0 & 52 & 26 & 44,54 & 7,79 \\
T1 & 52 & 32 & 42,54 & 6,44 \\
T2 & 51 & 28 & 42,04 & 5,55 \\
\hline
\end{tabular}

Fonte: Banco de dados da pesquisa

Legenda: Max: máximo, Min: mínimo, MD: média, DP: desvio padrão

Os resultados da FACT-F também foram associados, por meio do Teste Exato de Fisher, com as características sociodemográficas e em T0 apresentou relação significante com a escolaridade. As mulheres que possuíam entre seis e dez anos de estudos foram as que obtiveram os menores escores e sentiram menos fadiga.

Em T2, as variáveis menopausa e a prática de atividade física, apresentaram resultados significativos. As participantes que estavam na pré-menopausa antes de iniciar o tratamento quimioterápico, foram as que mais apresentaram fadiga após a última quimioterapia. Quanto à prática de atividade física, sete mulheres relataram praticar algum tipo de atividade, como caminhada e andar de bicicleta. Porém, dessas, apenas três praticavam de forma sistematizada com uma frequência semanal. Mesmo com esse número reduzido, as mulheres que praticavam 
Qualidade do sono e fadiga em mulheres com câncer de mama durante o tratamento quimioterápico | 10 alguma atividade física relataram menor percepção de fadiga, em comparação com as que não praticavam.

Não foi encontrada relação significativa entre o sono e a fadiga, porém, houve correlação entre a qualidade do sono e a fadiga do tipo negativo, indicando uma relação inversa entre as variáveis, ou seja, à medida que uma aumenta, a outra diminui. No meio do tratamento (T1) e após a última quimioterapia (T2), ocorreu uma correlação significativa de intensidade média entre as variáveis como demonstrado na Tabela 5.

Tabela 5- Distribuição das mulheres submetidas à quimioterapia para câncer de mama, segundo os escores médios e da correlação da Subescala Fadiga com o PSQI. Ribeirão Preto (SP), 2013.

\begin{tabular}{|c|c|c|c|c|}
\hline \multicolumn{5}{|c|}{ Subescala Fadiga } \\
\hline & & T0 & T1 & T2 \\
\hline$P S Q$ & no bom & 44,80 & 44,12 & 43,91 \\
\hline$P S Q$ & no ruim & 44,18 & 39,56 & 40,67 \\
\hline & le $p$ & 0,86 & 0,12 & 0,14 \\
\hline \multirow[t]{2}{*}{ Sono } & $\mathrm{R}$ & $-0,05^{*}$ & $-0,48^{*}$ & $-0,39^{*}$ \\
\hline & $\mathrm{r} \neq 0$ & 0,78 & 0,01 & 0,04 \\
\hline
\end{tabular}

Fonte: Banco de dados da pesquisa

Legenda: $\mathrm{p}<0,05$ pelo Teste de Mann Whitney

r- coeficiente de correlação de Spearman; r $\neq 0$ indica se o coeficiente de correlação de Spearman/ é significantemente diferente de zero

\section{Discussão}

Sabe-se que os distúrbios do sono em pacientes oncológicos podem ser uma condição pré-existente que se agrava com a doença ou como resultado do diagnóstico e da terapêutica proposta, e dentre os diversos tipos de cânceres, o de mama é o que apresenta maiores índices de alterações do sono. ${ }^{14}$ Essas alterações podem ser vinculadas à toxicidade quimioterápica sofrida pelo sistema nervoso central, que resultaria em alterações indiretas do sono, uma vez que as drogas quimioterápicas não possuem especificidade celular e atingem o tecido normal. ${ }^{15}$ 
11 I Silva PR, Lóris Cruz AP, Nascimento TG, Gozzo TO

No presente estudo, identificou-se que as participantes não apresentaram alterações quantitativas do sono, contudo, os problemas de início e manutenção do mesmo estão presentes e são caracterizados por despertares noturnos em decorrência de nictúria e ondas de calor, com frequência de três ou mais vezes na semana.

Esses achados são corroborados por estudo com 80 mulheres com câncer de mama que avaliou, prospectivamente, a qualidade do sono em três momentos, pré-quimioterapia, durante o tratamento e seis meses após seu término por meio do PSQI. Os resultados apontaram que os distúrbios mais relatados foram levantar várias vezes para ir ao banheiro e calores noturnos com aumento gradual entre os momentos e, consequentemente, mais episódios de fadiga (p<0,001). ${ }^{16}$

A fadiga relacionada ao câncer e o sono deficiente em mulheres com cancer de mama tem sido considerados reciprocos, tanto em amostras com diferentes tipos de câncer, quanto em estudos realizados somente com mulheres com câncer de mama durante a quimioterapia. ${ }^{17-18}$ Os resultados dessa pesquisa ainda são inconsistentes em apontar a fadiga relacionada ao câncer como um fator de risco para o sono ruim.

Entretanto, é possível observar que a ocorrência de fadiga piora a qualidade do sono e compromete a qualidade de vida, interferindo ainda nos sintomas depressivos. ${ }^{16}$, dados que confirmam a associação entre a qualidade do sono e a ocorrência de fadiga encontrados no presente estudo.

Destaca-se que há uma correlação negativa nos atuais achados, pois a medida que a qualidade do sono piora, os episódios de fadiga se tornam mais frequentes, o que pode ser justificada pela descrição das participantes ao apontarem a dificuldade de manutenção do sono. Esta relação de sintomas também foi observada em estudo que constatou alterações na qualidade do sono após o início da quimioterapia, destacando que o sono seria importante para lidar com outros eventos adversos como, por exemplo, a fadiga. ${ }^{3}$

A ocorrência de fadiga e alterações do sono em indivíduos com doenças oncológicas pode ser observada em estudos prévios. ${ }^{19-20}$ Outros estudos demonstram também que as mulheres com 
Qualidade do sono e fadiga em mulheres com câncer de mama durante o tratamento quimioterápico | 12 câncer de mama em tratamento quimioterápico apresentam fadiga e qualidade do sono ruim, em qualquer fase deste, seja antes de inicia-lo, no decorrer ou após seu encerramento.,6

A persistência dos sintomas de fadiga e das alterações do sono ao término da quimioterapia pode estar relacionada a fatores comportamentais, sociais e fisiológicos, advindos da sua experiência com a doença e preocupações com a saúde futura. O período de tempo varia de meses até cinco anos após o referido tratamento, entretanto, sem apresentar associação entre esses dois sintomas., ${ }^{4,21}$

O término do tratamento quimioterápico pode normalizar os padrões de sono e repouso para algumas mulheres, contudo, o sono noturno não reparador e a sonolência diurna podem persistir para outras. As perturbações do sono podem se tornar crônicas, especialmente se outras modalidades de tratamento forem recomendas após a quimioterapia. ${ }^{15}$

Outro aspecto observado no presente estudo e que merece destaque é a associação entre o status menopausal e a fadiga, sendo que as mulheres na pré-menopausa apresentaram maiores escores desta. Os fogachos podem ser um dos fatores contribuintes e/ou agravantes da fadiga e distúrbios do sono, como apontado nos resultados. A literatura aponta que os sintomas da menopausa, como as ondas de calor e os distúrbios de humor em decorrência das alterações hormonais, apresentam forte associação com a ocorrência de fadiga e alteração no padrão do sono. ${ }^{16,22,23}$ Sugere-se, assim, que a menopausa precoce induzida pela quimioterapia possa intensificar tais eventos adversos.

Além disso, foi possível identificar, de forma significante, que dois contextos contribuíram para o alívio da fadiga e para a melhora da qualidade do sono. Em relação a fadiga, identificou-se que a prática de atividade física auxiliou no seu manejo pós-tratamento. Estudos prévios com mulheres com câncer de mama corroboram com os achados do presente estudo, demonstrando que os exercícios físicos interferem positivamente no alívio da fadiga, além de contribuir na estruturação e na melhora da qualidade do sono, da insônia e sonolência excessiva. ${ }^{3,24}$ 
Quanto a associação entre a qualidade do sono e o estado civil, observou-se que as mulheres com companheiro apresentaram melhor qualidade do sono. Acredita-se que essa associação ocorra pelo apoio familiar que pode minimizar os níveis de ansiedade e medo. Como evidenciado em estudo com mulheres com câncer de mama que investigou a percepção destas sobre o apoio de seus companheiros as participantes perceberam o apoio dos parceiros de forma proativa durante o tratamento, mesmo quando eles não compreendiam o sofrimento causado pelos eventos adversos da terapêutica. ${ }^{25}$

É importante salientar o papel da equipe de saúde diante de condições que podem contribuir para a ocorrência de distúrbios do sono em mulheres com câncer de mama, como a nictúria, as ondas de calor, dor e a dificuldade de iniciar o sono devido ansiedade e medo. Tais condições são investigadas e conhecidas pelos profissionais de saúde, percebe-se, entretanto, que não recebem a devida atenção e acompanhamento terapêutico. Acredita-se que uma das justificativas para essa situação é a ausência de queixas por parte das mulheres e a falta de avaliação sistemática por parte da equipe de saúde.

Observa-se que muitos estudos realizados com essa temática são transversais, os quais não possibilitam identificar a relação de causa e efeito entre o sintoma e o câncer, fatos que destacam a importância de uma investigação anterior ao tratamento para avaliar a presença destes distúrbios e um possível manejo dos mesmos.

Tais dados justificam a pesquisa nessa área pois, associados ao aumento da vulnerabilidade dessa população específica, estão sujeitas ao efeito cumulativo dos quimioterápicos e ao impacto físico dos eventos adversos. Associado as estas questões estão as alterações nas atividades diárias, a ansiedade e o humor deprimido, os quais contribuem para a ocorrência das perturbações do sono, principalmente durante e após a quimioterapia.

\section{Conclusões}


Qualidade do sono e fadiga em mulheres com câncer de mama durante o tratamento quimioterápico | 14

Com o objetivo de identificar a ocorrência de mudanças no padrão de sono antes, durante e após o tratamento quimioterápico e correlacionar estes com episódios de fadiga em mulheres com câncer de mama, os resultados possibilitaram confirmar a sua ocorrência. As alterações no padrão do sono foram caracterizadas pela dificuldade de iniciar e manter o sono e pelos despertares noturnos durante todo o tratamento.

Além disso, verificou-se que há uma correlação entre a má qualidade do sono e a ocorrência de episódios de fadiga. Nota-se que os achados deste estudo apontam para o impacto da má qualidade do sono durante o tratamento e a importância de sua devida identificação, para promover manejo adequado, a fim de evitar episódios recorrentes e intensificação da fadiga. Os resultados também sugerem que os exercícios físicos e a presença de um companheiro podem contribuir no alívio de tais eventos adversos.

Neste sentido, enfatiza-se a atuação do profissional de saúde, especialmente do enfermeiro, na identificação e controle destes eventos. Acredita-se que orientações e manejo adequado dos sintomas como a fadiga e os distúrbios do sono pela equipe de enfermagem, são necessários e essenciais para alivio de desconfortos e melhora do bem-estar das mulheres em tratamento quimioterápico.

Vale ressaltar que o tamanho da amostra foi um fator limitante na realização do estudo. Entretanto, os resultados encontrados sugerem a realização de novos estudos, com amostras maiores, aumento na periodicidade das avaliações tanto do sono quanto da fadiga entre os ciclos de quimioterapia, além de avalições periódicas após o término do mesmo.

\section{Referências}

1. Ferreira RGR, Franco LFR. Efeitos colaterais decorrentes do tratamento quimioterápico no câncer de mama: revisão bibliográfica. Rev Univ Vale Rio Verde [Internet]. 2017 ago [acesso em 2018 abr 12];15(2):633-8.

Disponível em: http://periodicos.unincor.br/index.php/revistaunincor/article/view/3759/pdf_725 doi: http://dx.doi.org/10.5892/ruvrd.v15i2.3759. 
2. Chaoul A, Milbury K, Spelman A, Basen-Engquist K, Hall MH, Wei Q, et al. Randomized trial of Tibetan yoga in patients with breast cancer undergoing chemotherapy. Cancer [Internet]. 2018 jan [acesso em 2018 jun 26];124(1):36-45. Disponível em: https://onlinelibrary.wiley.com/doi/epdf/10.1002/cncr.30938

3. Amorim JR, Silva IA, Shimizu IS. Avaliação da qualidade do sono em pacientes com câncer de mama em quimioterapia. Rev Bras Mastologia [Internet]. 2017 set [acesso em 2018 abr 12];27(1):3-7. Disponível em: http:/www.rbmastologia.com.br/wp-content/uploads/2017/01/MAS-v27n1_3-7.pdf doi: 10.5327/Z201700010002RBM.

4. Lamino DA, Pimenta CAM, Braga PE, Mota DDCF. Fadiga clinicamente relevante em mulheres com câncer de mama: prevalência e fatores associados. Investig Enferm [Internet]. 2015 jan [acesso em 2018 jun 24];17(1):65-76. Disponível em: http://www.redalyc.org/html/1452/145233516005/ doi: https://doi.org/10.11144/Javeriana.IE17-1.fcrm

5. Fakih R, Rahal M, Hilal L, Dany M, Karam S, Shehab L, et al. Prevalence and severity of sleep disturbances among patients with early breast cancer. Indian J Palliat Care [Internet]. 2018 jan-mar [acesso em 2018 jun 14];24(1):35-8. Disponível em: https://www.ncbi.nlm.nih.gov/pmc/articles/PMC5801627/ doi: 10.4103/IJPC.IJPC_137_17

6. Levkovich I, Cohen M, Pollack S, Drumea K, Fried G. Cancer-related fatigue and depression in breast cancer patients postchemotherapy: different associations with optimism and stress appraisals. Palliat Support Care [Internet]. 2015 out [acesso em 2018 maio 02];13(5):1141-51. Disponível em: https://www.ncbi.nlm.nih.gov/pubmed/25201115 doi: 10.1017/S147895151400087X

7. Jim HSL, Jacobsen PB, Phillips KM, Wenham RM, Roberts W, Small BJ. Lagged relationships among sleep disturbance, fatigue, and depressed mood during chemotherapy. Health Psychol [Internet]. 2013 jul $\begin{array}{llllll}\text { [acesso em } 2018 \text { jun } & \text { 23];32(7):768-4. }\end{array}$ https://www.ncbi.nlm.nih.gov/pmc/articles/PMC3700563/ doi: 10.1037/a0031322

8.Whisenant M, Wong B, Mitchell SA, Beck SL, Mooney K. Distinct trajectories of fatigue and sleep disturbance in women receiving chemotherapy for breast cancer. Oncol Nurs Forum [Internet]. 2017 nov [acesso em 2018 jun 26];44(6):739-50. Disponível em: https://www.ncbi.nlm.nih.gov/pmc/articles/PMC5856248/pdf/nihms948252.pdf doi: 10.1188/17.ONF.739750

9. Siegel S, Castellan JRNJ. Estatística não paramétrica para ciências do comportamento. Porto Alegre (RS): Artmed; 2006.

10. Yellen SB, Cella DF, Webster K, Blendowski C, Kaplan E. Measuring fatigue and other anemiarelated symptoms with the Functional Assessment of Cancer Therapy (FACT) measurement system. J Pain Symptom Manage [Internet]. 1997 fev [acesso em 2018 abr 02];13(2):63-74. Disponível em: https://www.jpsmjournal.com/article/S0885-3924(96)00274-6/pdf 
Qualidade do sono e fadiga em mulheres com câncer de mama durante o tratamento quimioterápico | 16

11. Ishikawa NM, Thuler LCS, Giglio AG, Baldotto CSR, Andrade CJC, Derchain SFM. Reproducibility of Functional Assessment of Cancer Therapy-Fatigue (FACT-F) questionnaire for cancer patients. Appl Cancer Res [Internet]. 2008 [acesso em 2018 abr 02];28(2):55-61. Disponível em: https://www.researchgate.net/publication/266160631_Reproducibility_of_Functional_Assessment_of_Can cer_Therapy-_Fatigue_FACT-F_Questionnaire_for_Cancer_Patients

12. Buysse DJ, Reynolds CF, Monk TH, Berman SR, Kupfer DJ. The pittsburg sleep quality index: a new instrument for psychiatric practice and research. Psychiatr Res [Internet]. 1989 maio [acesso em $2018 \mathrm{abr}$ 05];28(2):193-213. Disponível em: https://linkinghub.elsevier.com/retrieve/pii/0165-1781(89)90047-4 doi: https://doi.org/10.1016/0165-1781(89)90047-4

13. Bertolazi NA, Fagondes SC, Hoff LS, Dartora EG, Miozzo IC, Barba MEF, et al. Validation of the Brazilian Portuguese version of the Pittsburgh Sleep Quality Index. Sleep Med [Internet]. 2011 jan [acesso em 2018 abr 05];12(1):70-5. Disponível em: https://www.sleep-journal.com/article/S13899457(10)00380-1/fulltext doi: http://dx.doi. org/10.1016/j.sleep.2010.04.020

14. Savard J, Ivers H, Savard MH, Morin CM. Cancer treatments and their side effects are associated with aggravation of insomnia: results of a longitudinal study. Cancer [Internet]. 2015 maio [acesso em 2018 maio 03];121(10):1703-11. Disponível em: https://onlinelibrary.wiley.com/doi/abs/10.1002/cncr.29244 doi: $10.1002 /$ cncr.29244

15. Harris B, Ross J, Sanchez-Reilly S. Sleeping in the arms of cancer: a review of sleeping disorders among patients with cancer. Cancer J [Internet]. 2014 set-out [acesso em 2018 maio 03];20(5):299-305. Disponível em: https://insights.ovid.com/pubmed?pmid=25299138 doi: 10.1097/PPO.0000000000000067

16. Sanford SD, Wagner LI, Beaumont JL, Butt Z, Sweet JJ, Cella D. Longitudinal prospective assessment of sleep quality: before, during, and after adjuvant chemotherapy for breast cancer. Support Care Cancer [Internet]. 2013 abr [acesso em 2018 out 12];21(4):959-67. Disponível em: https://link.springer.com/article/10.1007\%2Fs00520-012-1612-7 doi: 10.1007/s00520-012-1612-7

17. Tian L, Lin L, Li HL, Chen KJ, Zhang XJ, Qian SJ et al. Prevalence and associated factors of cancerrelated fatigue among cancer patients in Eastern China. Oncologist [Internet]. 2016 nov [acesso em 2018 out 15];21(11):1349-54. Disponível em: https://www.ncbi.nlm.nih.gov/pubmed/27449523/ doi:10.1634/theoncologist.2015-0537

18. Overcash J, Tan A, Patel K, Noonan A. Factors associated with poor sleep in older women diagnosed with breast cancer. Oncol Nurs Forum [Internet]. 2018 maio [acesso em 2018 out 15];45(3):359-71. Disponível em: https://www.ncbi.nlm.nih.gov/pubmed/29683125/ doi: 10.1188/18.ONF.359-371

19. Fisher WI, Johnson AK, Elkins GR, Otte JL, Burns DS, Yu M, et al. Risk factors, pathophysiology, and treatment of hot flashes in cancer. CA Cancer J Clin [Internet]. 2013 maio [acesso em 2018 maio 03];63(3):167-92. Disponível em: https:/www.ncbi.nlm.nih.gov/pmc/articles/PMC3640615/ doi: $10.3322 /$ caac. 21171 
20. Mansano-Schlosser TC, Ceolim MF. Fatores associados à má qualidade do sono em mulheres com câncer de mama. Rev Latinoam Enferm [Internet]. 2017 mar [acesso em 2018 maio 01];25:e2858. Disponível em: http://www.revistas.usp.br/rlae/article/view/130762 doi: 10.1590/1518-8345.1478.2858

21. Cheng H, Sit JWH, So WKW. The symptom burden in breast cancer survivors. Curr Breast Cancer Rep [Internet]. 2016 mar [acesso em 2018 abr 29]:8(1):40-6. Disponível em: https://link.springer.com/article/10.1007/s12609-016-0201-z doi: 10.1007/s12609-016-0201-z

22. Schmidt ME, Wiskemann J, Schneeweiss A, Potthoff K, Ulrich CM, Steindorf K. Determinants of physical, affective, and cognitive fatigue during breast cancer therapy and 12 months follow-up. Int $\mathrm{J}$ Cancer [Internet]. 2018 mar [acesso em 2018 maio 04];142(6):1148-57. Disponível em: https://onlinelibrary.wiley.com/doi/abs/10.1002/ijc.31138 doi: 10.1002/ijc.31138

23. Vincent AJ, Ranasinha S, Sayakhot P, Mansfield D, Teede HJ. Sleep difficulty mediates effects of vasomotor symptoms on mood in younger breast cancer survivors. Climacteric [Internet]. 2014 out [acesso em 2018 out 12];17(5):598-604. Disponível em: https://www.ncbi.nlm.nih.gov/pubmed/24673553 doi: $10.3109 / 13697137.2014 .900745$

24. Schmidt ME, Wiskemann J, Armbrust P, Schneeweiss A, Ulrich CM, Steindorf K. Effects of resistance exercise on fatigue and quality of life in breast cancer patients undergoing adjuvant chemotherapy: A randomized controlled trial. Int J Cancer [Internet]. 2015 jul [acesso em 2018 jun 24];137(2):471-80. Disponível em: https://onlinelibrary.wiley.com/doi/abs/10.1002/ijc.29383

25. Yamamoto S, Tazumi K, Arao H. Support not corresponding to transition to a new treatment: women's perceptions of support provided by their male partners during hormonal therapy. Int J Qual Stud Health Well-being [Internet]. 2015 [acesso em 2018 out 12];10(29283):1-9. Disponível em: https://doi.org/10.3402/qhw.v10.29283.

\section{Autor correspondente:}

Thais de Oliveira Gozzo

E-mail: thaisog@eerp.usp.br

Endereço: Avenida dos Bandeirantes, 3900 - Campus Universitário, Ribeirão Preto - SP, Brasil.

CEP: $14040-902$

\section{Contribuições de Autoria}

1 - Pamina Roberta da Silva

Concepção, planejamento do projeto de pesquisa, análise e interpretação dos dados.

2 - Lóris Aparecida Prado da Cruz

Análise e interpretação dos dados e revisão crítica.

3 - Talita Garcia do Nascimento

Rev. Enferm. UFSM, Santa Maria, v. 37, p. 1-18, 2019 
Qualidade do sono e fadiga em mulheres com câncer de mama durante o tratamento quimioterápico | 18 Análise e interpretação dos dados e revisão crítica.

4 - Thais de Oliveira Gozzo

Concepção, planejamento do projeto de pesquisa, análise e interpretação dos dados e aprovação final da versão para publicação.

\section{Como citar este artigo}

Silva PR, Lóris Cruz AP, Nascimento TG, Gozzo TO. Qualidade do sono e fadiga em mulheres com câncer de mama durante o tratamento quimioterápico. Rev. Enferm. UFSM. 2019 [Acesso em: 2019 jun 15];vol ex:p1-p18. DOI:https://doi.org/10.5902/2179769232732 